\title{
Human Identification and Fingerprints: A Review
}

\section{Nitin Kaushal ${ }^{1 *}$ and Purnima Kaushal ${ }^{2}$}

${ }^{1}$ Department of Oral and Maxillofacial Pathology ,B.R.S. Dental College and Hospital, Village Sultanpur,Panchkula Haryana, India

${ }^{2}$ Department of Anthropology, Panjab University,Chandigarh, India

\begin{abstract}
Fingerprints have been the gold standard for personal identification within the forensic community for more than one hundred years. Fingerprints and finger marks combine to provide the most powerful means of personal identification available to police and courts. The basic patterns of fingerprints are loops, whorls and arches that can be found in fingerprints. The standard methodology used by fingerprint experts to conduct friction ridge examinations is called ACE-V, for analysis, comparison, evaluation, and verification, which are the four fundamental phases utilized in this process. The recovered prints can be manually compared with known antemortem records or searched through an automated fingerprint system (AFIS) in order to verify or establish identity.
\end{abstract}

Keywords: Forensic; Antemortem; Fingerprint; Identification

\section{Introduction}

Fingerprints have been the gold standard for personal identification within the forensic community for more than one hundred years. The science of fingerprint identification has evolved over time from early use of fingerprints to mark business transactions in ancient Babylonia to their use today as core technology in biometric security devices and as scientific evidence in courts of law throughout the world. Fingerprints are the patterns formed by the raised papillary ridges on fingertips, which contain rows of pores that connect to sweat glands. After many years of research and study it has been established that papillary ridges on the fingers and palms of the hands, and the soles and toes of the feet, remain throughout life true to the mould in which they were fashioned by nature, unless their symmetry is permanently disturbed by some deep-seated injury [1]. These ridges, which are formed during the first few months of fetal life, not only remains immutable during lifetime, but after death seem to have the peculiar property of outlasting every other recognizable feature of the body.

Fingerprints and finger marks combine to provide the most powerful means of personal identification available to police and courts. How this became such a powerful tool arises from the realization that the patterns of ridge skin and their details are unique, immutable, universal, easy to classify, and leave marks on any object handled with bare hands. The understanding of the value of fingerprints led to research in detection techniques and to operational and strategic uses for fingerprints. Fingerprints form definite patterns that appear to have a general resemblance in shape and design. Their resemblance allowed fingerprint pioneer to device systems of classification for fingerprint patterns; now they allow practical use to be made of large collections of prints that can be filed according to definite scheme [2].

\section{Fingerprint Fundamentals}

The use of fingerprints in forensic science is based on several fundamental principles. The first is that, the probability of finding two people with identical fingerprints is very small. In fact, no two identical fingerprints have ever been found same. Galton calculated that probability of finding identical prints was 1 in 64 millions. A second principle is that an individual's fingerprints do not change with time. The pattern of ridges on a person's fingertips, palms and soles at birth remains unchanged until death. Consequently, a detective can be certain that a criminal's fingerprints will remain unchanged until death. Finally, there are enough similarities in the patterns of ridges on people's fingers that can be classified. The basic patterns of fingerprints are loops, whorls and arches that can be found in fingerprints. About 60 to 65 percent of the populations have loop patterns, 30 to 35 percent have whorls, and only about 5 percent have arches. The arches can be either plain or tented, and the whorls can be classified as central pocket, lateral pocket, twins and accidentals (Figure 1).

Detailed examination of the friction ridge skin also reveals that ridge path, in most instances, is not continuous across the entire surface of a finger. Some ridges, called ending ridges, will flow and abruptly come to an end, while other ridges, called dividing ridges or bifurcations, will flow and separate into two separate and distinct ridges. Additionally, some ridges are as long as they are wide and are called dots [3]. These ridge events are commonly referred to as characteristics or minutiae, and their spatial relationship to one another in a friction ridge impression is the basis for fingerprint comparison and identification. An arch has ridges that enter from one side of the pattern, make a wave in the middle, and pass out the opposite side from which they entered. In a whorl, the friction ridges tend to have a circular or spiral ridge flow. It is important for an examiner to note the ridge flow of a print for orientation purposes and the recognition of focal areas that will ultimately assist in the identification process. While pattern configuration alone cannot be used for individualization, it can be used for exclusionary decisions made by an examiner [3].

Fingerprints fall into three categories: latent, known, and plastic impressions. A latent fingerprint is the two-dimensional reproduction of the friction ridges of the finger on an object by means of perspiration, oils, or other contaminants that coat the surface of the ridges when a finger touches an item. These types of prints generally must be made visible through the use of forensic technology such as alternate light sources, chemical techniques, or fingerprint powders. In some instances, latent prints can be visualized without the use of any

${ }^{*}$ Corresponding author: Dr. Nitin Kaushal, House number 554, Sector 2, Panchkula - 134109, Haryana, Tel: 09780671752; E-mail: nitinzkaushal@yahoo. co.in

Received August 19, 2011; Accepted November 02, 2011; Published November 15,2011

Citation: Kaushal N, Kaushal P(2011) Human Identification and Fingerprints: A Review. J Biomet Biostat 2:123. doi:10.4172/2155-6180.1000123

Copyright: ( $) 2011$ Kaushal N, et al. This is an open-access article distributed under the terms of the Creative Commons Attribution License, which permits unrestricted use, distribution, and reproduction in any medium, provided the original author and source are credited. 


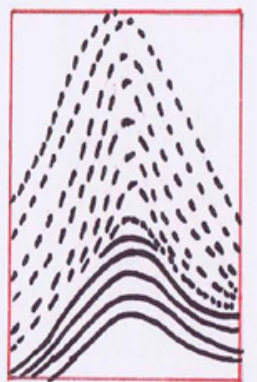

IA Simple Arch

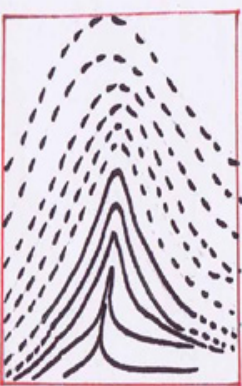

IB Tented Arch

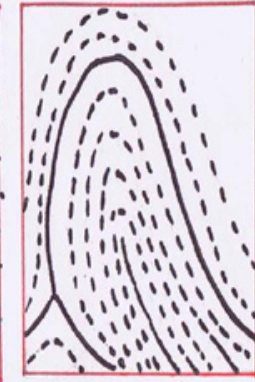

IC Loop Ulnar

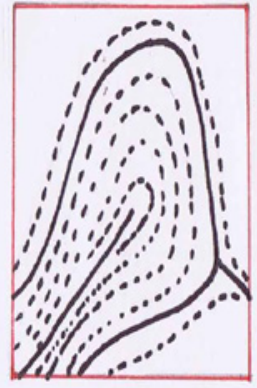

ID Loop Radial

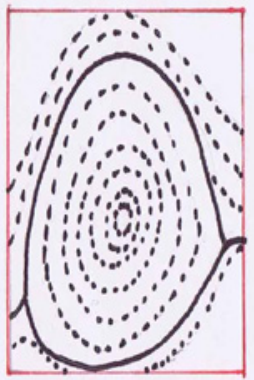

IE Simple Whorl

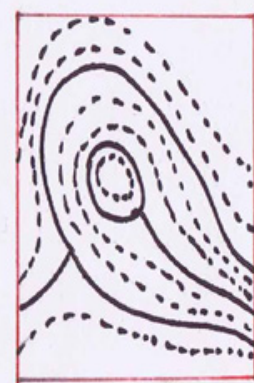

IF Central Pocket Loop

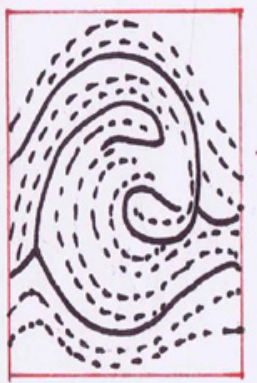

IG Twin Loop

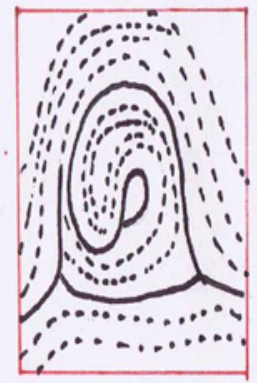

IH Accidental Whorl

Figure 1: Types of Finger Patterns: systematic drawings with boldly traced type lines

fingerprint processing techniques and are called patent prints. Latent impressions are deposited by chance and are usually fragmentary in nature with varying degrees of quality. Alternatively, a known fingerprint is the intentional reproduction of the friction ridges of the finger onto a fingerprint card or appropriate contrasting surface [4]. A known exemplar can be recorded using a number of standard techniques, to include black printer's ink, inkless/chemical methods, and Live scan, which is a computer-based system that creates digital fingerprint images by scanning the fingers. Finally, a plastic print is an impression left in a malleable substrate, such as wax or putty, which retains an image of the friction ridge arrangement [4]

\section{Fingerprint Ridge Density}

The ever increasing pattern of crime has made fingerprinting an indispensable tool in the hands of investigating officers. If the sex of the individual is established with certainty, the burden of the investigating officer would be reduced by half. In this context, sex based differences in fingerprint patterns and the density of the finger ridges becomes relevant. Sex differences in fingerprint ridge density are highly individualistic and forms the basis for personal identification in forensic examination these days. Recent studies have reported that females have a significantly higher ridge density than males $[5,6,7]$. The higher ridge density is female has been attributed to the level of ridge thickness and it is opined that females tend to have finer epidermal ridges details. Consequently, the females have significantly higher ridge density than male in a given area [8] . Jantz [9], found sex and race differences in finger ridge count correlations. Moore [11] mentions that females have finer epidermal ridge detail than males and Okajima [10] found fork index to be higher in female fingerprints when compared to males [6]. The degree of ridge densities can be used as a presumptive indicator of sex of an unkown print left at a crime scene [5,12].

\section{Comparison and Identification of Friction Ridge Patterns}

While the terminology used to describe the fingerprint identification process has varied over the years, the basic methodology employed by forensic examiners has remained relatively unchanged. One aspect that has changed, however, is an increased awareness of the underlying scientific basis for fingerprint identification. This change has resulted in the standardization of the identification process based on the extensive research of former Royal Canadian Mounted Police Staff Sergeant David R. Ashbaugh [13], which centers around a quantitativequalitative philosophy to fingerprint examination called ridgeology. Ridgeology is a holistic approach that focuses on the biological uniqueness of friction ridges and involves the sequential examination of the features and spatial relationship of ridges, noting the quality and quantity of the assessed information for identification purpose. This examination is conducted using a methodology that incorporates sound scientific protocols and practices, allowing for accurate and repeatable conclusions that meet rigorous scientific standards. The standard methodology used by fingerprint experts to conduct friction ridge 
examinations is called ACE- $\mathrm{V}$, for analysis, comparison, evaluation, and verification, which are the four fundamental phases utilized in this process.

Analysis focuses on the examination of the quantity and quality of information present in a print, which can be broken down into three levels of detail [14]. Level 1 detail refers to the overall ridge flow and pattern type of a print. Level 2 details refer to ridge path, which corresponds to the spatial relationship of ridges and their characteristics in a print. Level 3 detail refers to individual ridge attributes, which involve ridge shapes and pore structure/location in a print. The fingerprint examiner must consider various quality factors, such as distortion, that could alter the reliability of the observed information when determining the suitability of a print for comparison purposes. The information present in the latent or poorest quality print is always examined first, followed by examination of the known or best quality print.

Comparison of friction ridge impressions is a side-by-side assessment of the information analyzed in both prints [14]. The latent or poorest quality print is compared to the known or best quality print to minimize cognitive bias. The examiner first assesses the level 1 information from the analysis of the latent print and compares this with the information gathered from the analysis of the known print. If the information matches, the examiner then assesses the level 2 information from the analysis of the latent print and compares it with the information gathered from the analysis of the known print. Comparison is not a simple "point counting" exercise; in fact, there is no scientific basis for a minimum point threshold or specific number of characteristics that must match in two prints for an identification decision to be reached by an examiner [14]. Level 3 information is usually noted when assessing level 2 detail and, if visible, is compared as well. These comparative measurements begin at a focal point selected by the examiner and progress through the ridges of the entire print in series. For information to match in both the prints, the ridge path should have adequate quality, clarity, relative position and have the same unit relationship. Identification decision can be reached only if the latent and the known's prints are in agreement [14]. Due to the pliability of the friction skin, and other environmental factors, friction ridge impressions of the same finger will never look exactly alike.

Evaluation involves rendering a decision based on the results of the analysis and comparison phases of the identification process. There are three possible conclusions that can be reached by an examiner as defined by the Scientific Working Group on Friction Ridge Analysis Study and Technology (SWGFAST) [15]. The first conclusion is individualization (identification) and involves the determination that sufficient information present in two impressions matches, meaning that they are from the same source. The second conclusion is exclusion and is the determination that the information present in two impressions does not match, meaning that they are not from the same source. The third conclusion is an inconclusive decision and is the determination that a conclusive comparison cannot be reached because of a lack of quality or absence of a comparable area in the known exemplar.

Verification is the final step in the ACE-V methodology. Although verification is not technically part of the identification process, it serves as a form of peer review, ensuring reliable and accurate results [15]. All individualizations made by a fingerprint expert are verified, through an independent examination of the identified prints, by a second qualified latent print examiner as a quality assurance mechanism. Verification of exclusion or inconclusive decisions also can be performed but is not required by SWGFAST. Blind verification which is a rigorous type of pear review is being used by FBI, in which the evaluation decision made by the original finger analyst is not disclosed to the verifying examiner. For fingerprints to be used as a means of individualization there are two basic premises which also form the basics of friction ridge identification process [15]. These premises concern the individuality and persistency of the friction skin, which have been scientifically validated over time through academic research and the work of experts in the field of fingerprints.

Individuality refers to the fact that fingerprints are unique; no two areas of friction ridge skin are the same, not even on identical twins. The basis for this statement rests in human embryology and genetics, beginning during fetal development. The physiology of friction ridge skin begins with the development of the volar pads, which are protuberances of tissue that begin to form on the tips of the fingers at about the eighth week of gestation. The degree of complexity of the volar pads (their size, shape, and location on the finger) greatly influences ridge flow or level 1 detail [15]. These volar pads regress or area absorbed back into the finger at about the tenth or eleventh week of gestation, when friction ridges begin to form. Primary ridges develop first, followed by secondary ridge development or the occurrence of furrows between the papillary ridges. Random development of friction ridges and their level 2 and level 3 details, results from the infinite number of environmental factors, even though most of these activities have a genetic component [15]. The end result of these genetic and environmental variances during friction ridge formation is complete biological uniqueness, down to the structure of a single ridge.

Persistency refers to the fact that friction ridges are permanent and remain constant throughout a person's lifetime, until decomposition after death, unless otherwise affected by accidental injury or intentional mutilation. The basis for this statement rests in human anatomy and the histology of the skin. As the body sloughs off dead skin cells, they are replaced by new skin cells generated from the bottom or basal layer of the epidermis [15]. The cells joined together through cell junctions are replaced the same way for an entire lifetime unless scarring occurs. Thus, the basal layer acts as an immutable root system that is the foundation for the permanency of friction ridges and their corresponding level 1,2, and 3 detail.

\section{Automated fingerprint identification technology}

While the recovery of identifiable postmortem impressions from human remains is an integral part of the forensic identification process, it is imperative that these impressions be compared with an antemortem standard in order to have any value in establishing or verifying human identity. The expeditious identification of postmortem remains depends on the most important technological advancement in the history of fingerprinting: the Automated Fingerprint Identification System. This computer system, known as AFIS, has evolved from its early use as a means of searching criminal ten- print records to its use today in identifying suspects of crimes through latent print searches against local, state, and national fingerprint repositories. Biometric data bases of millions of fingerprint cards along with the records of criminal history submitted by law enforcement agencies around the country constituted Integrated Automated Fingerprint Identification System (IAFIS), which was released by FBI in 1999 [16]. IAFIS allows the FBI 
and other criminal justice agencies to electronically access the national fingerprint repository in Clarksburg, West Virginia, for ten-print and latent print searches, meaning that criminals can be tracked by their fingerprints throughout the United States. There is a probability that the individuals who are arrested have their fingerprint records in the FBI criminal master file (CMF). For a job or for a military service the individuals might have been fingerprinted as a part of a background investigation and their records are contained in the FBI civil file (CVL) [16].

Some of the most important criteria in using fingerprints as a means of human identification are the cost-effective and rapid reporting of results, which is directly related to fingerprint computer technology. AFIS, in addition to being a crime-fighting tool, is also instrumental in the identification of the dead [16]. If a dog tag or wallet can be obtained from decedents, the fingerprint record can be located by entering personal identifying information from these items into AFIS and printing off the antemortem record if it exists. The postmortem prints then can be compared manually to the antemortem record to verify identity. In instances of closed-population disaster situations, meaning that the identities of individuals killed in the event are readily known, personal identifying information can be obtained from items such as an airline manifest and entered into AFIS to retrieve fingerprint records. The records can be obtained and manually compared with recovered postmortem impressions, depending on the number of fatalities.

Larger disasters often will preclude quick manual comparison of antemortem records, which means that postmortem prints must be searched electronically through AFIS. Postmortem prints are first scanned into AFIS and encoded, meaning that the friction ridge minutiae or characteristics are digitized. Criteria such as pattern type and finger position are then selected followed by the launch of the fingerprint search. Searches of postmortem impressions can take only a few minutes, depending on the submitted criteria, and result in a list of candidates with the closest correlation to the submitted print. Although the I in AFIS represents identification, the comparison of the candidates and any identification decision, as it relates to latent print examination, is made by a certified fingerprint examiner and not the computer. The FBI has portable IAFIS terminals that can be deployed to disaster scenes around the world with the capability of searching recovered postmortem impressions through remote access to the national fingerprint repository.

In open-population disasters, meaning that the identities of individuals killed in the event are not readily known, recovered postmortem prints should be searched through an automated fingerprint system for identification purposes. This is best addressed by a practical look at the deployment of AFIS and the use of fingerprints for mass fatality victim identification in the aftermath of the 2004 South Asian Tsunami in Thailand. Over five thousand people were killed when tsunami waves struck the coast of Thailand on December 26,2004 . Because Thailand is a popular vacation destination, the dead included not only local residents but also many tourists, particularly from Scandinavian countries. The magnitude of the disaster resulted in a worldwide request for antemortem identification records for those believed killed in the catastrophe. As a result, AFIS was established to assist in the massive identification effort because no automated fingerprint system existed in Thailand. Fingerprint cards submitted by various government agencies, as well as latent prints developed on items believed to have been handled by the deceased, were entered into AFIS and used as antemortem standards.

Identifiable postmortem fingerprints, recovered from the majority of the bodies using the boiling technique, were then searched against the available antemortem database, resulting in numerous identifications. An important issue discovered in Thailand when using an automated fingerprint system for victim identification involved dimensional variations associated with recovered postmortem impressions. In some instances, the friction skin will expand or shrink to a point that the abnormal size of the recovered prints must be addressed by the examiner in order for a correlation to be made with an antemortem record in AFIS. The lack of antemortem fingerprint records, especially in developing countries, and the ability to recover quality postmortem impressions can limit the effectiveness of fingerprints in identifying the dead [16]

\section{Validity of fingerprint identification}

The validity of forensic fingerprint evidence has been challenged by academics, judges and the media. While fingerprint identification was an improvement on earlier anthropometric systems, the subjective nature of matching, despite a very low error rate, has made this forensic practice controversial. Certain specific criticisms are now being accepted by some leaders of the forensic fingerprint community, providing an incentive to improve training and procedures.

Criticism: The words "reliability" and "validity" have specific meanings to the scientific community [17]. Reliability means that successive tests bring the same results. Validity means that these results are judged to accurately reflect the external criteria being measured. Although experts are often more comfortable relying on their instincts, this reliance does not always translate into superior predictive ability. For example, in the popular Analysis, Comparison, Evaluation, and Verification (ACE-V) paradigm for fingerprint identification, the verification stage, in which a second examiner confirms the assessment of the original examiner, may increase the consistency of the assessments. But while the verification stage has implications for the reliability of latent print comparisons, it does not assure their validity [18]. The few tests that have been made of the validity of forensic fingerprinting have not been supportive of the method.

Despite the absence of objective standards, scientific validation, and adequate statistical studies, a natural question to ask is how well fingerprint examiners actually perform. Proficiency tests do not validate a procedure per se, but they can provide some insight into error rates. In 1995, the Collaborative Testing Service (CTS) administered a proficiency test that, for the first time, was "designed, assembled, and reviewed" by the International Association for Identification (IAI).The results were disappointing. Four suspect cards with prints of all ten fingers were provided together with seven latents. Of 156 people taking the test, only 68 (44\%) correctly classified all seven latents. Overall, the tests contained a total of 48 incorrect identifications. David Grieve, the editor of the Journal of Forensic Identification, describes the reaction of the forensic community to the results of the CTS test as ranging from "shock to disbelief," and added: "Errors of this magnitude within a discipline singularly admired and respected for its touted absolute certainty as an identification process have produced chilling and mind- numbing realities. Thirty-four participants, an incredible $22 \%$ of those involved, substituted presumed but false certainty for truth. By any measure, this represents a profile of practice that is unacceptable and thus demands positive action by the entire community'. What is striking about these comments is that they do not come from a critic of the fingerprint community, but from the editor of one of its premier publications. Investigations have been conducted into whether experts can objectively focus on feature information in fingerprints without being misled by extraneous information, such as context [17]. 
Fingerprints that have previously been examined and assessed by latent print experts to make a positive identification of suspects have then been re-presented to those same experts in a new context which makes it likely that there will be no match. Within this new context, most of the fingerprint experts made different judgments, thus contradicting their own previous identification decisions [9]. Complaints have been made that there have been no published, peer-reviewed studies directly examining the extent to which people can correctly match fingerprints to one another [19]. Experiments have been carried out using naïve undergraduates to match images of fingerprints. The results of these experiments demonstrate that people can identify fingerprints quite well, and that matching accuracy can vary as a function of both source finger type and image similarity [19].

\section{Conclusion}

Fingerprint identification is the oldest forensic discipline known to man. Fingerprints have proved over time to be the most rapid, reliable, and cost-effective means by which to identify unknown deceased individuals, especially in a mass disaster setting. The recovered prints can be manually compared with known antemortem records or searched through an automated fingerprint system (AFIS) in order to verify or establish identity. The identification of remains through fingerprints accomplishes the most important and difficult mission of the forensic identification operation: the timely and accurate notification of families regarding the fate of their loved one [20].

\section{References}

1. Galton F (1892) Fingerprints. London: Macmillan \& Co.

2. Herschel WJ (1916) The origin of fingerprints. London: Oxford University Press.

3. Henry ER (1922) Classification and uses of fingerprints. (5th edn), London: HM Stationary Office.

4. Browne DG, Brock (1954) A Fingerprints: Fifty years of scientific crime detection. New York: EP Dutton \& Co.

5. Acree MA (1999) Is there a gender difference in fingerprint ridge density? Forensic Sci Int 162: 35-44.
6. Nayak VC, Rastogi P, Kanchan T, Lobo SW, Yoganarsinhma K, et al. (2010) Sex differences from fingerprints ridge density in the Indian population. J Forensic Leg Med 17: 84-86.

7. Gutierrez-Redomero E, Alonso C, Romero E, Galera V (2008) Variability of fingerprint ridge density in a sample of Spanish Caucasians and its application to sex determination. Forensic Sci Int 180: 17-22.

8. Krishan K, Ghosh A, kanchan T, Ngangom C, Sen J (2010) Sex differences in fingerprint ridge density-causes and further observation. J Forensic Leg Med 17: 172-173

9. Jantz RL (1977) Sex and race differences in finger ridge-Count correlations Am J Phy Anthropol 46:171-176.

10. Okajima M (1970) Frequency of fork in epidermal ridge minutiae in fingerprint Am J Phy Anthropol 32: 41-48.

11. Moore RT (1994) Automatic fingerprint identification systems: In Lee HC Gaensslen, RE editors, Advances in fingerprint technology. Boca Raton, Florida: CRC Press 169.

12. Nithin MD, Balaraj BM, Manjunatha B, Shashidhar CM (2009) Study of fingerprint classification and their gender distribution among South Indian Population. J Forensic Leg Med 16: 460-463.

13. Ashbaugh DR (1999) Quantitative and qualitative friction ridge analysis: An introduction to basic and advanced ridgeology. Boca Raton, FL: CRC Press LLC.

14. Symposium (1995) Report: Israel National Police: International symposium on Fingerprint Detection and identification. J for Ident 45: 578-584.

15. Wertheim K, Maceo A (2002) The critical stage of friction ridge and pattern formation. J for Ident 52: 35-85

16. Wilder HH, Wentworth B Personal identification. Boston: Gorham Press 1918.

17. Dror IE, Charlton P, Peron AE (2006) Contextual information renders experts vulnerable to making erroneous identifications. Forensic Science International 156: 74-78.

18. Snady LZ (2005) Fingerprint evidence. L Law \& Policy 143: 13-20.

19. Vokey JR, Tangen JM, Cole SA (2009) On the preliminary psychophysics of fingerprint identification. Quart J Exp Psycho 62: 1023-1040.

20. Senn DR, Stimson PG (2010) Forensic Dentistry. New York: CRC Press. 